\title{
Enhanced P2P Services Providing Multimedia Content
}

\author{
E. Ardizzone, L. Gatani, M. La Cascia, G. Lo Re, and M. Ortolani \\ Dipartimento di Ingegneria Informatica (DINFO), Università degli Studi di Palermo, Viale delle Scienze, Ed. 6, \\ 90128 Palermo, Italy \\ Received 30 January 2007; Accepted 28 August 2007 \\ Recommended by Yi Cui

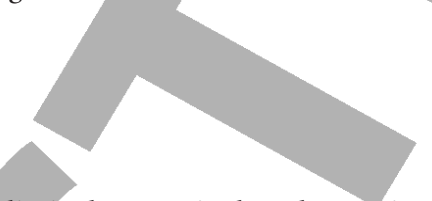

The retrieval facilities of most Peer-to-Peer (P2P) systems are limited to queries based on unique identifiers or small sets of keywords. Unfortunately, this approach is very inadequate and inefficient when a huge amount of multimedia resources is shared. To address this major limitation, we propose an original image and video sharing system, in which a user is able to interactively search interesting resources by means of content-based image and video retrieval techniques. In order to limit the network traffic load, maximizing the usefulness of each peer contacted in the query process, we also propose the adoption of an adaptive overlay routing algorithm, exploiting compact representations of the multimedia resources shared by each peer. Experimental results confirm the validity of the proposed approach, that is capable of dynamically adapting the network topology to peer interests, on the basis of query interactions among users.

Copyright (c) 2007 E. Ardizzone et al. This is an open access article distributed under the Creative Commons Attribution License, which permits unrestricted use, distribution, and reproduction in any medium, provided the original work is properly cited.

\section{INTRODUCTION}

Peer-to-Peer (P2P) networks are distributed systems in which each node runs software with equivalent functionality, in order to operate without requiring central coordination [1]. The P2P paradigm has emerged in the past few years, mainly due to file sharing systems such as Napster [2] and Gnutella [3]. In the research community there has been an intense interest in designing and studying P2P systems. Due to their decentralization, these systems promise improved robustness and scalability.

It is well known that consumers are gathering more and more digital multimedia contents. Consumers capture contents using their digital cameras, digital camcorders, and mobile phones; and store them on different devices. They nowadays tend to store their data in such quantity, as it is becoming increasingly difficult for them to manage, to find and, in the end, to enjoy the videos and images they create.

While the amount of multimedia content keeps growing, locating and obtaining the desired resource has become a difficult task. Traditionally, P2P users request resources using keywords, or simply by searching for a specific file name pattern. This approach is insufficient when the collected data are huge or distributed, especially in the P2P environment. Namely, users might annotate the same file with different file names and keywords, making the data location process errorprone and user-dependent; moreover, artificial intelligence technologies are not mature enough to provide a complete automatic annotation solution bridging the semantic meanings and the low-level descriptors.

In this paper, we present a framework for sharing multimedia resources in a P2P network, exploiting an automatic content-based approach. The present work is based on previous research [4], and represents an extended version which includes a more detailed description of the resource selection mechanism, and a wider set of experiments.

With respect to current content-based image retrieval (CBIR) systems, we envisage the potential use of P2P networks in both scattering data storage and distributing workload of feature extraction and indexing. Through the realization of CBIR in P2P networks, enormous image collections can be managed without installing high-end equipment by the exploitation of individual user's contribution. Furthermore, we make use of the computational power of peers for image preprocessing and indexing in addition to data storage.

One of the most challenging problems related to datasharing P2P systems is content localization. It determines whether the system resources can be efficiently used or not, affecting the scalability and robustness of P2P systems. Therefore, in order to effectively exploit the potential of CBIR in P2P networks, we also propose an adaptive mechanism for query routing that can well balance the storage overhead and the network load. 
The rest of the paper is structured as follows. Section 2 presents both a brief review on the existing CBIR techniques, and the video descriptors chosen for representing multimedia resources; Section 3 illustrates the proposed approach to storing, managing, and retrieving multimedia content; some experimental results are presented in Section 4, and finally we conclude with summary remarks in Section 5.

\section{RELATED WORK}

Although the lookup of multimedia data in P2P networks represents a new, interesting research field known as CBP2PIR (content-based peer-to-peer image retrieval), to the best of our knowledge, only few works exist where this problem is addressed. In this section, we outline some ideas previously presented in relevant literature that are related to the key aspects of our work.

\subsection{P2P for multimedia content management}

In [5], the Firework Query Model for CBP2PIR is proposed. The main idea is to cluster peers with similar resources, using the set of feature vectors, as signature value of a peer, in order to measure similarity. The Firework Query Model exploits two classes of links (normal random links and privileged attractive links) in order to route queries. A query starts off as a Gnutella-like flooding query. If a peer deems the query too far away from the peers local cluster centroid, it will forward it via a random link, decreasing the query Time-To-Live (TTL). Otherwise, it will process the query, and forward it via all its attractive links without decreasing the TTL.

A similar CBIR scheme for P2P networks, based on compact peer data summaries, is presented in [6]. To obtain the compact representation of a peer's collection, a global clustering of the data is calculated in a distributed manner. After that, each peer publishes how many of its images fall into each cluster. These cluster frequencies are then used by the querying peer to contact only those peers that have the largest number of images present in one cluster given by the query.

In [7], the authors investigate a CBIR system with automated relevance feedback (ARF) using nonlinear Gaussianshaped radial basis function and semisupervised selforganizing tree map clustering technique. The authors apply the CBIR system over P2P networks by grouping the peers into community neighborhoods according to common interest.

In [8], a different overlay setup technique is introduced, in order to cluster peers according to the semantic and feature-based characteristics of their multimedia content.

Finally, Wu et al. [9] propose a local adaptive routing algorithm that dynamically modify the network topology toward a small-world structure, using a learning scheme similar to that considered in this paper. However, they design their protocol with the aim of supporting an alternative model for peer-based Web search, where the scalability limitations of centralized search engines can be overcome via distributed Web crawling and searching.
Our work belongs to the same research area of the abovementioned proposals, but introduces a novel combination of video indexing and retrieval techniques, with a $\mathrm{P} 2 \mathrm{P}$ adaptive routing strategy capable of leading to dynamically emerging small-world network communities.

\subsection{Extracting information from multimedia content}

Representing and describing digital image and video content has been an area of active research since mid 1990s, when this topic came into strong attention of both research and industry people working in the developing areas of CBIR, digital libraries, image and video coding, web query engines, and so on. The nature of the problem is well known: visual information is provided with intrinsic semantics that is very hard or impossible to make explicit by a manual description, such as a list of keywords or a full-text description. Thus, a number of content-based algorithms and techniques have been developed in the last decade (see [10] for a review) to make possible the automatic or semiautomatic extraction of lowor medium-level feature descriptors from images and videos, mainly in the areas of indexing and retrieval of image and video databases and of video coding.

CBIR video representation may be based on the decomposition of the video sequence into short video units named shots [11] or, more recently, into video objects [12]. Shot clustering has been proposed to represent video scenes. A shot cluster is considered a scene; a hierarchical scene transition graph, that is, a collection of directed graphs may be then used to model the whole video structure.

Simple shot content representations may be obtained through the description of few representative frames. A limited number of frames (said r-frames or key-frames) are selected from each shot, and each $\mathrm{r}$-frame is therefore described in terms of its visual content, for example, through color and texture descriptors. Thus the description problem is reconducted to the extraction of static descriptors [11]. Motion activity may be also taken into account, for example, by computing motion features related to short sequences in which r-frames are embedded. In this way, a dynamic description of the $\mathrm{r}$-frame may also be obtained.

The r-frame selection and the computation of visual and motion features may be performed in a number of ways. In the following, we will refer to images to indicate either single images, in the case of still image applications, or representative frames, that is, frames representing a subpart of a video sequence in the case of video applications.

In order to correctly classify and represent video contents it is fundamental to discover similarities in the extracted images; and the known useful features for this purpose are color and texture. In the last years, several color-based techniques have been proposed for video annotation (e.g., region-based dominant color descriptors [13], multiresolution histograms [14], and vector-quantized color histograms [15]). Several texture descriptors have also been proposed that try to mimic the human similarity concept, but they are normally useful only in classifying homogeneous texture. Generic images usually contain different kinds of texture, so that a global 
texture descriptor hardly may describe the content of the whole image.

In this paper, we adopted HSV color histogram, edge density, motion magnitude, and motion direction histogram to represent $r$-frames. These features have been chosen as to lead to satisfactory results in previous work [16].

\section{THE PROPOSED APPROACH}

The technique we propose in this paper tries to improve the scalability and efficiency of the resource discovery in the unstructured $\mathrm{P} 2 \mathrm{P}$ environment, through an adaptive routing algorithm which suppresses flooding.

Queries issued by a user are routed to neighbor peers in the overlay network, in order to find resources that satisfy them. Initially the network has a random, unstructured topology (each peer is assigned a number of neighbors randomly chosen), and queries are forwarded as in the scoped flood model. However, we adopt an approach that dynamically selects the neighbors to which a query has to be sent or forwarded. The selection process is performed with the aim to detect peers that with high probability share resources satisfying the query. The selection is driven by an adaptive learning algorithm by which each peer exploits the results of previous interactions with its neighbors to build and refine a model (profile) of the other peers, describing their interests and contents. Each peer is characterized by one (or several) general interest and shares resources according to its interest. The characteristics of each peer are summarized in a peer profile. When an agent needs to forward a query, it compares the query with its known profiles, in order to rank all known peers and select the best suited to return good response. The network topology (i.e., the actual set of peers that are neighbors in the overlay) is then dynamically modified on the basis of the learned contexts and the current information needs, and the query is consequently routed according to the predicted match with other peers' resources.

\subsection{Multimedia content representation}

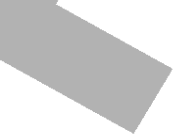

As outlined in Section 2, the choice of r-frames is a crucial task in automatic video annotation. In this work, we adopted a simplified technique of nonlinear time sampling, based on the comparison of a cumulative difference of frame brightness values with a threshold, whose value has to be tuned experimentally [16]

In our approach, a video descriptor is structured in a hierarchical way. At the highest level, this descriptor simply consists of references to the shot descriptor for each shot belonging to the video; each of those shot descriptors, in turn, consists of: (i) the shot duration (in seconds), (ii) the number of $r$-frames contained in the shot, and (iii) a reference to the $r$-frame descriptor for each $r$-frame belonging to the shot. R-frames (or still images) are globally described, and the relative visual descriptor consists of attributes of both static and motion-based kind. The former kind is based on texture and color, whereas the latter is based on the optical flow field of the r-frame; their computation involves considering a few frames before and after the $\mathrm{r}$-frame.

A simple but effective method [16], based on a 3-dimensional quantized color histogram in the "Hue-SaturationValue" (HSV) color space and a Euclidean metric, is used here to compare the query image to images contained in the database. The HSV quantization needed to compute a discrete color histogram is done taking into account that hue is the perceptually most significant feature. Thus a finest quantization has been used for hue, allowing for 18 steps, whilst only 3 levels are allowed for saturation and value. In such a way, we obtain a $162(18 \times 3 \times 3)$ bins HSV histogram, that may be easily represented by a $162 \times 1$ vector.

The texture features we propose are related to coarseness, directionality, and position of texture within the image. All these features are based on edge density measures. Edge density is directly related to coarseness, directionality is addressed by repeating the edge measure for different directions, and spatial position is taken into account by a simple partitioning of the r-frame. In particular, we first subdivide the r-frame into four equal regions. For each region, we compute the edge maps through directional masks, respectively, aligned along the directions 0, 45, 90, and 135 degrees. Values of edge map exceeding a fixed threshold are considered edge pixels. The threshold value has been determined experimentally. The ratio between the number of edge pixels and the total number of pixels is the edge density. Since we determine 4 edge density values for each region, we have a $16 \times 1$ texture-based vector.

Motion-based descriptors are based on the optical flow field [17] of the r-frame, and their computation involves considering a few frames before and after the $r$-frame. We used a gradient-based technique and the second-order derivatives to measure optical flow [16]. The basic measurements are integrated using a global smoothness constraint. This technique allows to obtain a dense and sufficiently precise flow field at a reasonable computational cost. Once the optical flow field is computed, we need a method able to code the associated information in a form adequate for content description. First, we segment the flow field into four equal region; for each region we then compute motion based features. The splitting was performed to preserve spatially related information that are not integrated in the computed features. In conclusion, the adopted motion descriptors are a measure of the average motion magnitude in the considered region, and a normalized 18 bins histogram of motion vectors directions.

In summary, the visual descriptor of an r-frame, computed automatically by the system, is a 254-dimensional vector $\underline{x}=\left[\begin{array}{llll}\underline{c} & \underline{t} & m & d\end{array}\right]$ where $\underline{c}$ is a 162-dimensional vector representing the global HSV color histogram and $\underline{t}=\left[\begin{array}{llll}t_{t l} & \underline{t_{t r}} & \underline{t_{b l}} & \underline{t_{b r}}\end{array}\right]$ is a 16-dimensional vector representing the edge density computed, respectively, over the topleft, top-right, bottom-left, and bottom-right quadrants of the r-frame. Similarly, $\underline{m}=\left[\begin{array}{llll}m_{t l} & m_{t r} & m_{b l} & m_{b r}\end{array}\right]$ and $\underline{d}=$ $\left[\begin{array}{llll}d_{t l} & \underline{d_{t r}} & d_{b l} & d_{b r}\end{array}\right]$ are a 4 -dimensional vector and a 72 dimensional vector containing, respectively, the average motion magnitudes and the 18 bins motion vectors direction histograms computed over the four regions as above. 


\subsection{The adaptive search algorithm}

Since our goal is to allow peers to form communities in a fully distributed way, they should find new peers and evaluate their quality in relation to their own interests. In our system, we follow an approach similar to the one presented in [9]. When a peer enters the network for the first time, the bootstrap protocol returns the address of some existing peers to get started. The new peer can then discover other nodes through these known peers. In the proposed system, a peer would discover new peers through its current neighbors, during the normal handling of queries and responses. Each peer maintains a fixed number of slots for known peers. This number can vary among peers depending on their available memory (a peer must properly prune other peers' information when needed). For each known peer, a profile which concisely describes the shared resources is stored. The actual set of neighbors, that is, those to whom queries are sent, is selected dynamically for each query at time step $t$ among all the known peers. In particular, when a peer receives a locally generated query, it compares the query with its stored profiles. Each peer applies a simple ranking algorithm for dynamically selecting peers to which query must be sent. The maximum number of selected peers depends on peer bandwidth and computational power to process neighbor data. Network connectivity is not negatively affected by dynamic neighbor selection, since each peer also stores references to other potential neighbors that may be used to replace previously selected ones, in case they are no longer available; nevertheless this is not per se a guarantee against network partitioning.

The selection mechanism tends to reinforce connections among peers with similar content; such nodes are thus clustered together in the overlay network, whereas fewer intercluster connections are maintained. This scenario resembles the typical small-world configuration [18], where each node in a network is reachable in a limited number of hops; namely, peers that share common interests should be directly connected, whereas peers sending queries out of their interest area will have their messages traverse several clusters before reaching the potential recipient. This is favorable in terms of effectiveness of the search procedure, but is known to be prone to network partitioning as consequence of the potential disconnection of one of the peers that act as hubs among clusters. A peer might thus not be able to get a successful query hit although the desired content is in fact available in the network, because it may be unreachable due to partitioning.

We propose here a heuristic for lessening the impact of this issue. Besides relying on the neighbors whose profiles show a commonality of interests, peers participating to our framework will additionally choose to forward queries to randomly selected nodes according to a small prefixed probability $P_{1}$. In order to do this, they must have previously stored profiles of all peers that they may have come in contact with, regardless of the similarity metric; this mechanism will guarantee higher variance to the profile list by allowing also "unlikely" peers to be selected once in a while. Moreover if, during the query forwarding step, the ranking pro- cedure cannot return any peer with a sufficiently high similarity measure (according to a predefined threshold), a peer will again choose a random neighbor, with a probability $P_{2}$, where $P_{2}>P_{1}$.

Although no strict guarantee can be provided against network partitioning, careful choice of the above-mentioned thresholds will make this phenomenon unlikely to occur.

Each peer profile maintains a concise representation of the shared resources, by the adoption of different techniques for textual and visual contents. In particular, the system adopts a simple taxonomy and Bloom filters [19] to build a binary vector that represents the textual contents. As regards visual resources, after the meaningful features have been extracted from the image database, each peer will work on extracting representative information that may succinctly describe its whole content. Finally, the set of cluster representatives may be used as a sort of "signature" for the content of each peer and we use their vectorial representation as reported at the end of Section 3.1.

Our system supports a basic query language (where a query string is interpreted as a conjunction of keys) for textual information retrieval, while a standard "queryby-example" approach is exploited to search the image database. When asked with a query, the system looks up the information in its profile database (using the selection mechanism described in Section 3.3) in order to obtain a list of candidate peers that might store data matching the query. When a peer receives a query from another peer, it checks its local repository in order to locate the resources that better match with the desired content. In particular, textual resources are searched using a standard keyword-based technique, while the visual similarity between two images is computed by means of a weighted sum of normalized Euclidean distances, as already presented in [16]. In order to normalize the distances, we estimate a probability distribution for the Euclidean distances of each visual feature (color, texture, motion), comparing each $r$-frame in a training database with all the others. These distributions are then used to normalize all the distances to the range $[0,1]$. The normalization is needed to make the use of a weighted sum of distances meaningful. Furthermore, a peer that has received a query can forward it to those neighbors whose profiles match the query. To this aim, the peer uses the same selection algorithm applied to locally generated queries (note that the peer automatically excludes both the peer that has forwarded the query, and the peer that has generated the query). In order to prevent potential DoS attacks which exploit the response system, we impose that a peer replies to a forwarded query sending the response to the neighbor that has forwarded the query, and not directly to the originating peer. To limit congestion and loops in the network, queries contain a TTL, which is decreased at each forward, and queries will not be forwarded when TTL reaches 0 . When a peer receives the responses for a locally generated query, it can start the actual resource downloading. Moreover, if a peer that has sent a response is not yet included in the list of known peers, a profile request is generated. For this request, the two peers contact each other directly. When the message containing the profile will arrive, the new peer will be inserted among the set of 


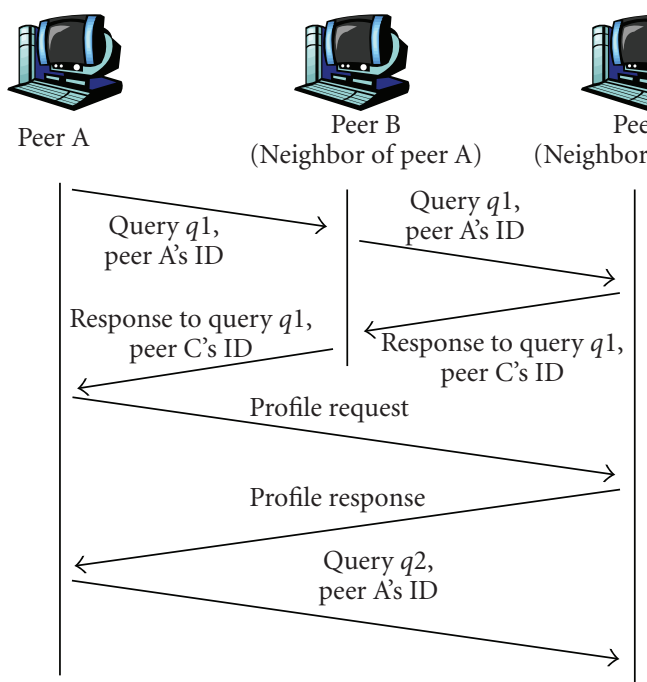

Figure 1: The process of neighbor discovery.

known peers and its features will be considered in order to select actual neighbors for the following queries (see Figure 1). The maintained profiles are continuously updated according to the peer interactions during the normal system functioning (i.e., matches between queries and responses). Moreover, a peer can directly request a more up-to-date profile if necessary.

\subsection{The selection mechanism}

As already mentioned, our system supports different query languages for textual information retrieval, and for image and video retrieval, respectively. In particular, for the latter kind of resources we employ a query-by-example paradigm for color and texture features and a direct query paradigm for motion features. In practice, the user presents to the system an image similar to the one she is looking for and, in the case of video query, provides information about motion (e.g., a zoom-in shot has a particular motion direction histogram, an almost-static shot has a very low motion magnitude, etc.). The technique we used for querying has already been presented in [16] and makes use of a weighted sum of normalized Euclidean distances. More specifically, in order to normalize those distances, we estimate a probability distribution for the Euclidean distances of each visual feature (color, texture, motion), comparing each $r$-frame in a training database with all the others. These distributions are then used to normalize all the distances to the range [0-1].

The similarity between the current query and the general interests of each peer is also managed differently on the basis of the kind of searched resource. Similarity between textual resources (as well as textual annotations and highlevel descriptors associated to multimedia resources) is evaluated exploiting a standard technique for textual retrieval. As regards visual resources, the peer computes the distance to each cluster representative and chooses the closest ones as possible matches. Furthermore, if the resources are opportunely indexed, the system can also exploit the representa- tion of the resources by means of Bloom filters [19] which are maintained into the peer profiles. This way, it is possible to check, with high probability, if a given resource belongs to the resource set shared by a peer. This approach enhances the topological properties of the emergent overlay network and it is very useful in those applications where resources are uniquely characterized by an identifier or are semantically annotated.

The selection mechanism takes primarily into account the experience that peers acquire during their normal interactions: each newly available piece of information is opportunely elaborated and exploited to enrich the system knowledge. Each peer profile maintains a concise representation of the shared resources, by the adoption of different techniques for textual and visual contents. In particular, the system adopts simple taxonomies and Bloom filters to build a binary vector that represents the textual contents. As regards visual resources, after the meaningful features have been extracted from the image database as described in Section 2.2, each peer will work on extracting representative information that may succinctly describe its whole content.

Once the contents have been properly represented as vectors in the search space, our implementation makes use of clustering techniques from literature through which each peer will roughly partition its data space into separate regions that represent different groups of related images. Specifically, we employ the well-known $k$-means clustering method presented for the first time in [20], whose underlying idea consists in assigning each data element to one cluster by means of a similarity function, which is often based on the Euclidean distance as a metric; each cluster is then represented by a prototype, or cluster representative, typically computed as the cluster centroid. The basic formulation of the algorithm assumes that the number of clusters is known in advance, which may be a too tight constraint for our present scenario; however, this requirement may be partially loosened with the use of controlled iterations and of a cluster validity assessment technique [21, 22]. Furthermore, in order to cope with the stream of continuously incoming data, we use a variation on the basic $k$-means algorithm that allows online updating of the computed clusters. Without delving into too much detail, our method builds upon the ideas presented in [23] and generalizes them from the binary case to multiclass classification. Finally, the set of cluster representatives may be used as a sort of "signature" for the content of each peer and will be spread to all nodes of the P2P network in order for them to be able to perform their searches. Another node that is presented with a request for a new element and needs to find out a list of possible owner candidates will compute the distance to each cluster representative and choose the closest ones as possible matches. It is worth noting that, while all processing is performed locally, manipulated objects exist in a globally defined vector space; hence all feature vectors, as well as all cluster centroids, are globally comparable; however, clusters are not required to have a global semantic validity as they are only used to compute relative distances.

After the peer has extracted representative descriptors for the content to be searched, it will compare them with its neighbors' profiles in order to find the best matches. 


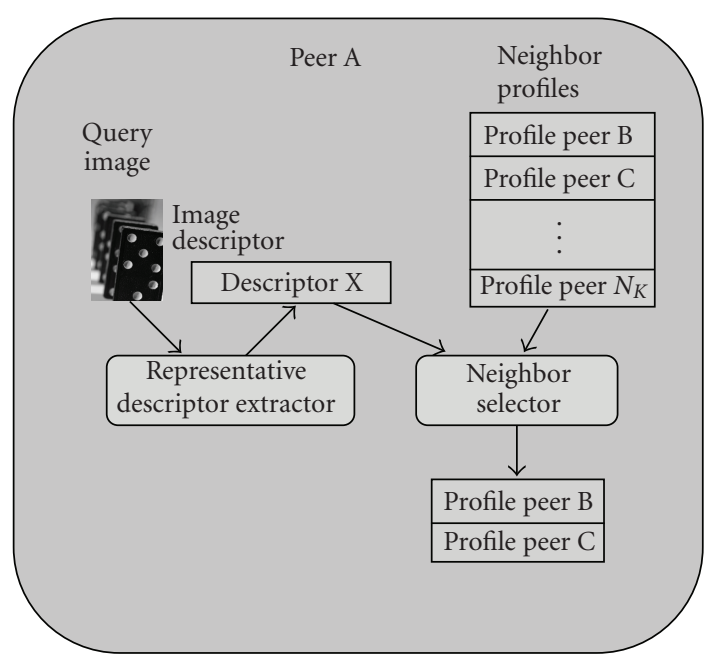

(a) The local selection process

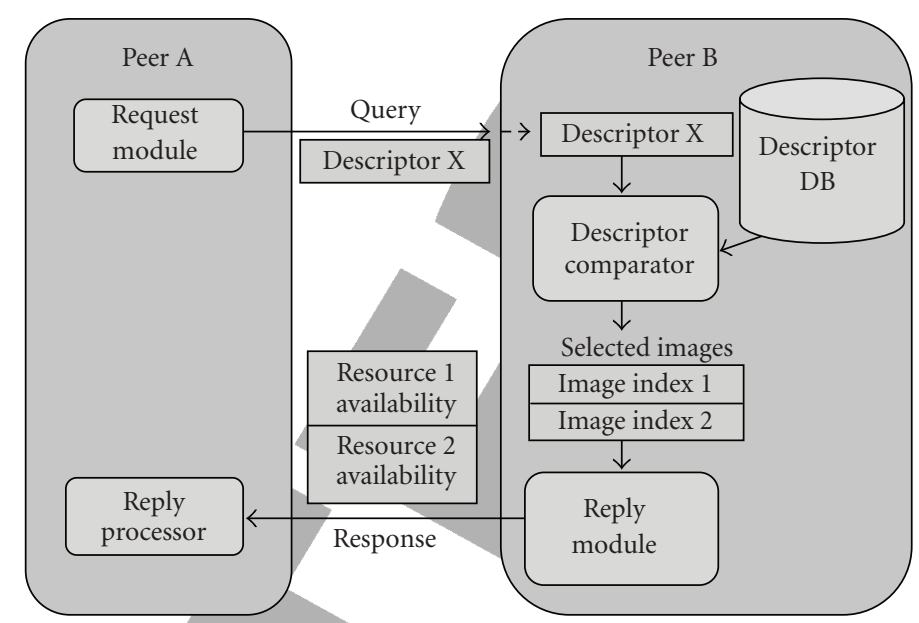

(b) Resource selection on remote node through descriptors

FIgURE 2: The resource selection mechanism.

TABLE 1: Selection criteria.

\begin{tabular}{lll}
\hline Parameter & Description & Weight \\
$\bar{R}_{t}$ & Current estimate of the contact & $\alpha$ \\
$R_{t-1}$ & Old reliability value of the contact (according to past history) & $1-\alpha$ \\
$R_{t}$ & New reliability value used to rank contacts & $\beta$ \\
$J$ & Percentage of contact interests with respect to query topics & $\gamma$ \\
$S$ & Percentage of successes provided by the contact & $\delta$ \\
$B$ & Result of membership test (produced by Bloom filter), if applicable \\
$C$ & Capability summarization of the contact (bandwidth, CPU, storage, etc.) \\
\hline
\end{tabular}

A basic criterion for conveniently selecting peers satisfying a given request would exploit the experience of past interactions among peers, thus giving a good indication about the probability that a contact could directly provide the resources searched. We enhance this criterion by adopting a further mechanism capable of singling out peers that, although not directly owning the desired resources, can provide good references to the resource owners. It is worth noting that while the first criterion, based on the commonality of interests, tries to increase the overlay network clusterization by the creation of intracluster links, the second one typically sets links between different clusters, providing a quick access to peers that are close to several resources. Furthermore, the selection mechanism considers some additional criteria, in terms of peer capabilities (bandwidth, CPU, storage, etc.) and end-to-end latency, in order to take into account the topological characteristics of the peer community (thus reducing the mismatch between the overlay and the real topology). Figure 2 visually describes the selection process, showing both the operations performed locally by a peer, and the interactions between that peer and a possible selected neighbor; this selected node would be the one that provides proper references in order to decide on the eventual request of resources.
In order to get a deeper understanding of the process just outlined, we can consider a formalized version. Each peer stores a parameter, $R$, associated to each of its contacts that provides a measure of that contact's reliability. The parameter value is related to the interactions in the peer community and it changes according to the criteria previously described (see also Table 1). Each single criterion gives a partial value. These partial values are then jointly considered by means of a weighted average (1) that produces an estimate of the overall reliability for the situation at the current timestep $t$. Indicating this estimate by $\bar{R}_{t}$, this yields:

$$
\bar{R}_{t}=\beta \cdot J+\gamma \cdot S+\delta \cdot B+\epsilon \cdot Q+\zeta \cdot(1-C),
$$

where

$$
\begin{gathered}
\beta+\gamma+\delta+\epsilon+\zeta=1 \\
0 \leq \beta, \gamma, \delta, \epsilon, \zeta \leq 1
\end{gathered}
$$

The current estimate $\bar{R}_{t}$ is finally combined with the old estimate $R_{t-1}$, generating the new value $R_{t}$ for the reliability parameter. In order to smooth the results of the selection process, a kind of time window is employed to balance new information against past experience. The new estimate is then formally computed by the formula:

$$
R_{t}=\alpha \cdot \bar{R}_{t}+(1-\alpha) \cdot R_{t-1},
$$


where

$$
0 \leq \alpha \leq 1, \quad \alpha \ll(1-\alpha) .
$$

The $R_{t}$ value is then exploited to rank all the known peers, according to the estimated reliability. In order to establish a balance between the exploration and exploitation of the search space, the algorithm in the early steps can select peers different from the top ones. This random search behavior is characterized by a probability of choosing no optimal contacts:

$$
P=\exp \left(\frac{\delta R_{t}}{T}\right),
$$

where, using an approach similar to that adopted in the "simulated annealing" searching technique [24], $\delta R_{t}$ represents the decrease in the reliability value, and the "temperature" $T$ is a control parameter.

\section{EXPERIMENTAL EVALUATION}

The experimental results presented in this section are aimed to validate the two main different aspects dealt with in the paper: namely, the adaptive routing mechanism adopted by the $\mathrm{P} 2 \mathrm{P}$ protocol and the image video descriptors proposed for multimedia content retrieval.

Regarding the first topic, the key idea of our approach is that a clustered overlay topology (where peers with shared interests and domains tend to form strongly connected communities) can spontaneously emerge by means of an intelligent peer collaboration. In particular, the adoption of our adaptive mechanism, based on a reinforcement learning scheme, should better cope with highly dynamic P2P communities, leading to topologies with small-world properties [18]. In such a topology, a flood-based routing mechanism (with limited scope) is well suited, since it allows any two peers to reach each other via a short path, while granting higher communication efficiency within clustered peer communities. Furthermore, the proposed approach should take advantage from the adaptive overlay rearrangement, in order to well cope with high node volatility and massive node disconnections.

Regarding the issue of multimedia content representation, we aim to validate the effectiveness of the adopted visual descriptors, in order to confirm the expected retrieval capabilities of our approach. In particular, the experimental evaluation should prove that the visual descriptors we adopted can encode the visual content reasonably well.

\subsection{Experimental settings}

We decided to use simulation to investigate the properties of the proposed approach. This is mainly due to the difficulty to obtain a precise and comprehensive snapshot of actually deployed P2P networks [25-27], whose behavior is complicated by the complex dynamics in overlay connections and peer lifetimes. Simulation of P2P networks can instead provide a straightforward and effective tool to conduct thorough analysis of their characteristics. In order to study the behavior of peer interactions in our system, we designed and implemented a simulator (see, also, [28]) that allows to model synthetic peer topologies, the shared resources, and the issued queries. The main goal of our simulator is to analyze the effectiveness of our routing protocol and the topology properties of emergent peer networks. As observed in [29], unstructured P2P systems are characterized by high temporal locality of queries (i.e., with high probability a single peer issues similar queries over time). Therefore, in the simulations carried out, each peer belongs to one or more groups of interest, according to the topics of owned resources and issued query. In order to better investigate how the adaptive mechanism proposed can support efficient resource searching, we consider that each peer generates queries belonging, with high probability, to one of the topics (however, a smaller number of queries is generated on a randomly selected topics). It is also worth noting that peers can have interests that partially overlap each other, and that each resource can be replicated on several peers.

Let $G=(V, E)$ denote a connected graph modeling a communications network, $N=|V|$ the cardinality of the set of vertices, and $d(i, j)$ the-length (in hops) of the shortest path between two vertices $i$ and $j$ in $V$. For the experimental analysis of emergent topological properties, we consider two network metrics, the clustering coefficient, $C(G)$, and the characteristic path length, $L(G)$, that well characterize the topological properties of dynamic networks.

The characteristic path length $L(G)$ is defined as the number of edges in the shortest path between two vertices, averaged overall pairs of vertices. To define the clustering coefficient $C(G)$, suppose that a vertex $v \in V$ has $k_{v}$ neighbors; then at most $k_{v}\left(k_{v}-1\right) / 2$ edges can exist between them (this occurs when every neighbor of $v$ is connected at every other neighbor of $v$ ). Let $C_{v}$, the local clustering coefficient of $v$, denote the fraction of these allowable edges that actually exist. The (overall) clustering coefficient is then defined as the average of $C_{v}$ over all $v$. While $L$ measures the typical separation between two vertices in the graph (a global property), $C$ measures the cliquishness (degree of compactness) of a typical neighborhood (a local property). Since in our simulations it is possible that the network is not always strongly connected, we adopt an alternative definition $\left(L^{\prime}(G)\right)$ for the characteristic path length, using the harmonic mean of shortest paths that can be computed irrespective of whether the network is connected:

$$
L^{\prime}(G)=\left(\frac{N}{N-1} \sum_{i, j \in V} d(i, j)^{-1}\right)^{-1} .
$$

We also compute the ratio $C / L^{\prime}$ that gives a good insight of the overall topological properties: high values are associated with networks that present both a strong clusterization, and a low average separation between nodes. To compute these metrics, the simulator takes a snapshot of the network for each time step: $C$ and $L^{\prime}$ are then computed in the directed graph which models the overlay network, based on each peer's actual neighbors.

Furthermore, in order to quantify the efficiency of the approach proposed, three further metrics are adopted: the query hit rate, HR, that represents the percentage of queries successfully replied, the query coverage rate CR, that 
represents the average number of nodes reached by a query, and the node message-load ML, that represents the average number of messages that a node has to process during a single time step.

Finally, to assess the effectiveness of the adopted multimedia content descriptor in the context of content-based retrieval, we use a normalized version of precision and recall that embodies the position in which relevant items appear in the retrieval list [30]. All the tests are performed using a database containing about $1500 \mathrm{r}$-frames obtained from about 500 shots. We consider 20 r-frames randomically chosen and we evaluate for each one of them the system response to a query by example. Recall and precision measurements require to determine which $r$-frames are relevant with respect to a posed query, but stating relevance is a very subjective task as also noted in [31]. To overcome this problem we adopt a subjective criterion: candidate-to-relevance r-frames for each query are determined by four different people (not including the authors) and a $\mathrm{r}$-frame is considered as relevant if at least three people chose it. Once known the correct query result we are able to evaluate performances. Fixed to $n$ the number of images to be retrieved, for each query we perform the following measures:

(i) AVRR, the average rank of all relevant, retrieved images;

(ii) IAVRR, the ideal average rank, that is, when all the relevant images are ranked at the top;

(iii) MT, the number of relevant images that are missed;

(iv) AVRR/IAVRR

In particular, let $I$ denote the number of relevant images among the $n$ retrieved, $\rho_{r}$ the rank of the $r$ th relevant retrieved image, and $T$ the total number of images relevant to the posed query. Then we can state the following definitions:

$$
\mathrm{AVRR}=\frac{1}{I} \sum_{r=1}^{I} \rho_{r} ;
$$

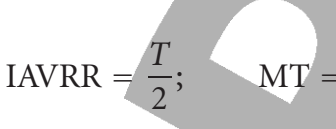

$=\frac{I}{T}$

Note that these measures depend on $n$, the number of retrieved images: in our experiments we perform each query twice, one for $n=32$ and one for $n=64$.

The visual descriptors we adopted, despite their compactness and the availability of simple algorithms to compute, have been proved to encode the visual content reasonably well. In particular, previous experiments [16] showed that visual descriptors are adequate in most cases if the image collections are not too large (less than 10,000 images). For larger image collection, when query results obtained using only visual descriptor tends to become unreliable, the use of textual information greatly improve the results.

Further investigation on our CBP2PIR system using both textual, and visual data showed very promising retrieval capability, confirming the feasibility of our searching method for feature vectors derived from multimedia resources.

\subsection{Experimental results}

In order to thoroughly evaluate the proposed approach, we preliminarily investigated the effectiveness of the techniques
TABLE 2: Average query results for twenty test queries by color and texture, with $n=32$ and $n=64$.

\begin{tabular}{clcc}
\hline & Metric & Color & Texture \\
\hline \multirow{5}{*}{$n=32$} & AVRR & 6.935 & 11.124 \\
& IAVRR & 5.501 & 10.836 \\
& AVRR/IAVRR & 1.270 & 1.063 \\
& MT & 0.854 & 0.474 \\
\hline \multirow{5}{*}{$n=64$} & AVRR & 10.074 & 21.996 \\
& IAVRR & 5.507 & 10.834 \\
& AVRR/IAVRR & 1.736 & 2.083 \\
& MT & 0.912 & 0.709 \\
\hline \multirow{3}{*}{} & & & \\
& & &
\end{tabular}

adopted for multimedia content representation. Then, we performed extensive simulations on the $\mathrm{P} 2 \mathrm{P}$ routing algorithm, considering several scenarios, each of them characterized by the variation of one significant simulation parameter. For each simulation, the aim is to study how network properties and searching performance change when the parameter value is varied. Finally, we analyzed the impact of dynamic changes in the peer communities, in order to test the robustness of the algorithm against such events. Since the initial random topology can affect the final results, for each simulation, we perform several independent simulations, averaging across all the results.

\subsubsection{Studying the effectiveness of multimedia content descriptors}

In order to assess the retrieval capabilities of the visual descriptors, we carried out several experiments, under different assumptions. As already mentioned, extensive results evaluating the retrieval performance based only on visual information have been reported [16]; in this paper we present some recall and precision measurements for the improved CBP2PIR framework. In particular, we report in Table 2 the average values of AVRR, IAVRR, AVRR/IAVRR, and MT related to 20 test queries (both color-based, and texture-based), respectively for $n=32$ and $n=64$. Results show that color indexing exhibits a distinctly good behavior.

\subsubsection{Studying the P2P searching mechanism}

For the sake of brevity, we can only present some representative results; a detailed performance evaluation of the proposed searching approach can be found in [28], confirming the idea that adaptive routing can properly work and that small-world network topologies can emerge spontaneously from local interactions between peers, structuring the overlay in such a way that it is possible both to locate information stored at any random node by only a small number of hops and to find quality resources quickly and even under heavy demands. In order to illustrate how the proposed approach can significantly improve searching performance in $\mathrm{P} 2 \mathrm{P}$ communities, we only report in Table 3 a comparison between the results obtained in the same experimental conditions by the adoption, respectively, of the algorithm proposed, a flooding scheme, and a random-walk searching 
TABLE 3: Comparison between adaptive routing, flooding, and random-walk.

\begin{tabular}{|c|c|c|c|c|c|c|c|}
\hline Searching scheme & Sim & $C$ & $L^{\prime}$ & $C / L^{\prime}$ & $\operatorname{HR}(\%)$ & $\mathrm{CR}$ & ML \\
\hline \multirow{3}{*}{ Adaptive routing } & Sim 1 & 0.184750 & 7.442001 & 0.024825 & 0.615112 & 28.193076 & 0.252483 \\
\hline & $\operatorname{Sim} 2$ & 0.185430 & 7.224156 & 0.025668 & 0.637082 & 28.308287 & 0.274244 \\
\hline & $\operatorname{Sim} 3$ & 0.188139 & 7.734175 & 0.024326 & 0.610095 & 27.471692 & 0.224489 \\
\hline \multirow{3}{*}{ Flooding } & Sim 1 & 0.004210 & 5.397369 & 0.000780 & 0.472454 & 22.459130 & 0.226549 \\
\hline & $\operatorname{Sim} 2$ & 0.004732 & 5.417398 & 0.000873 & 0.439865 & 22.828022 & 0.262706 \\
\hline & $\operatorname{Sim} 3$ & 0.003982 & 5.398215 & 0.000737 & 0.457117 & 22.408658 & 0.215307 \\
\hline \multirow{3}{*}{ Random-walk } & $\operatorname{Sim} 1$ & 0.002037 & 69.540365 & 0.000029 & 0.481486 & 33.870043 & 0.703346 \\
\hline & $\operatorname{Sim} 2$ & 0.002096 & 69.670214 & 0.000030 & 0.486615 & 32.933378 & 0.805104 \\
\hline & $\operatorname{Sim} 3$ & 0.002148 & 70.736614 & 0.000030 & 0.480732 & 32.168622 & 1.019697 \\
\hline & & \multicolumn{3}{|c|}{ Initial topology } & \multicolumn{3}{|c|}{ Final topology } \\
\hline Searching scheme & $\operatorname{Sim}$ & $C$ & $L^{\prime}$ & $C / L^{\prime}$ & $\mathrm{C}$ & $L^{\prime}$ & $C / L^{\prime}$ \\
\hline \multirow{3}{*}{ Adaptive routing } & $\operatorname{Sim} 1$ & 0.004210 & 5.397369 & 0.000780 & 0.184750 & 7.442001 & 0.024825 \\
\hline & $\operatorname{Sim} 2$ & 0.004732 & 5.417398 & 0.000873 & 0.185430 & 7.224156 & 0.025668 \\
\hline & $\operatorname{Sim} 3$ & 0.003982 & 5.398215 & 0.000737 & 0.188139 & 7.734175 & 0.024326 \\
\hline \multirow{3}{*}{ Flooding } & Sim 1 & 0.004210 & 5.397369 & 0.000780 & 0.004210 & 5.397369 & 0.000780 \\
\hline & $\operatorname{Sim} 2$ & 0.004732 & 5.417398 & 0.000873 & 0.004732 & 5.417398 & 0.000873 \\
\hline & $\operatorname{Sim} 3$ & 0.003982 & 5.398215 & 0.000737 & 0.003982 & 5.398215 & 0.000737 \\
\hline
\end{tabular}

mechanism. All the values related to clustering coefficient and characteristics path length are measured at the final time step of the simulation. It is worth noting that the adaptive algorithm performs significantly better both in term of success rate, and as regard the message load imposed on the network. Table 4 shows the $C$ and $L^{\prime}$ values both at the beginning, and at the end of the simulation, for the algorithm proposed and for flood-based search. It is straightforward to note that while flooding does not affect the topological properties, the approach proposed, although it starts from the same initial graph, achieves a final configuration where the topological metrics assume values that are typical of small-world networks.

\subsubsection{Studying the impact of dynamic changes in the peer community}

We analyze here the behavior of the system when the peer community is subject to a large amount of connection and disconnection events. All the simulations are carried out starting from the same initial conditions (considering 5000 timesteps for each execution). Since the considered scenarios are qualified by the event sequences, which depend from the adopted event models, in the following we present the results according to this categorization. Simulation results in Figure 3 are characterized both by a dynamic community, and a fixed number of participants; the peer number is maintained constant, since each connection is associated to a corresponding disconnection, according to a Pareto distribution for the model of events.

The following simulations consider a dynamic community with a variable number of participants. As reported in Table 5, six different cases are considered, each characterized by a synthetically predefined sequence of events: we believe that these choices well describe the plausible evolutions of $\mathrm{P} 2 \mathrm{P}$ communities. For each sequence, the number of peers at the initial and final steps and the total amount used in each simulation are also reported in Table 5.

For the sake of brevity, we only report the plots relative to the two most challenging cases, corresponding to the $3 \mathrm{rd}$ and 6th event sequence. A very high activity distinguishes the third synthetic sequence (see Figure 4), where many new connections and disconnections raise the peer expansion to the $65 \%$ of their initial value. Similarly, a relevant variability qualifies the sixth sequence (see Figure 5) which is also characterized by an unbalanced ratio between few peer additions and many disconnections. This behavior leads to a $37 \%$ reduction of the initial peer population.

As all the gathered data show, both algorithm properties (capability of evolving toward a small-world topology and efficiency) are minimally affected by the dynamic events, also when those involve large portions of the peer community (see Figures 4 and 5), thus confirming the robustness of the algorithm.

\section{CONCLUSION}

This paper presented a CBIR approach to information retrieval in P2P networks, that relies on an adaptive technique for routing queries and is specifically targeted to multimedia content search. The main motivation behind our work is that the huge amounts of data, their peculiar nature, and, finally, the lack of a centralized index make it particularly difficult to pursue the goal of efficiency in this kind of systems. Our approach employs a decentralized architecture which fully exploits the storage and computation capability of computers 


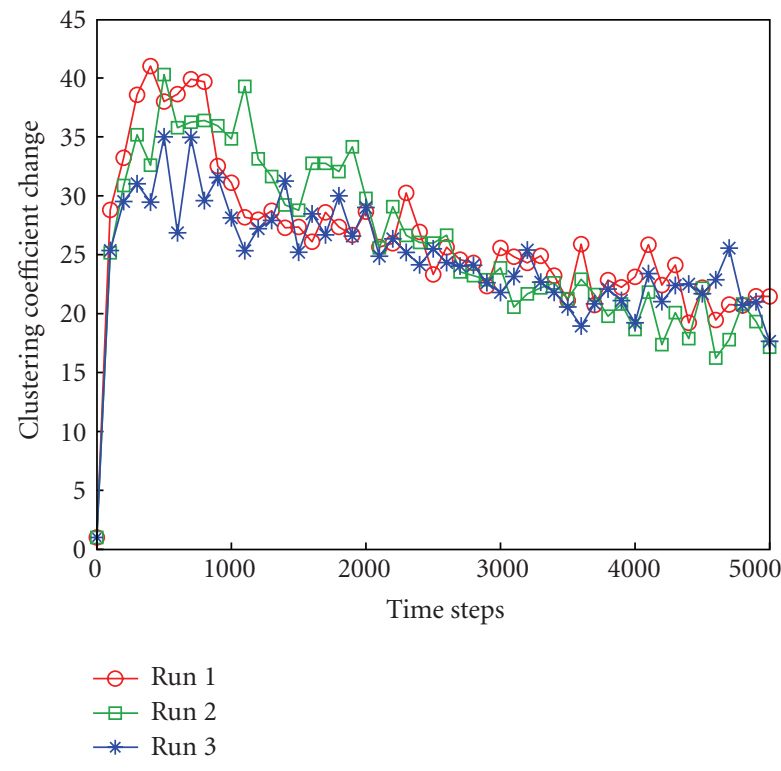

(a)

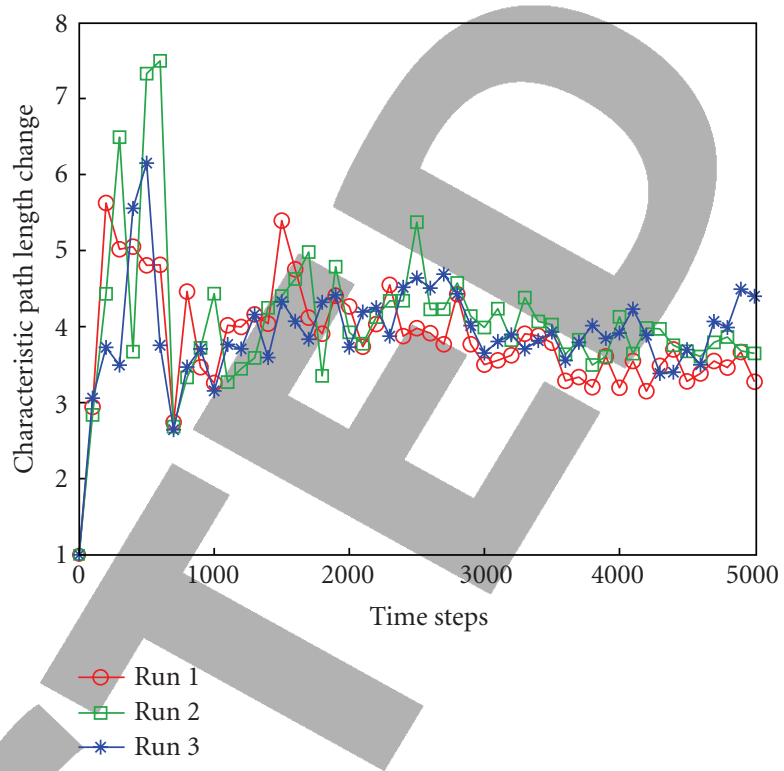

(b)

FIgURE 3: Relative variation of topological metrics using Pareto event model.

TABLE 5: Synthetic sequences for dynamic evolution of P2P communities.

\begin{tabular}{cccccc}
\hline Seq. & Initial peer no. & Final peer no. & Total peer no. & Kind of dynamic operations & Degree of dynamic evolution \\
\hline 1st & 500 & 550 & 550 & Only connections & Low increase \\
2nd & 500 & 575 & 611 & Connections and disconnections & Low increase \\
3rd & 500 & 825 & 890 & Connections and disconnections & High increase \\
4th & 500 & 450 & 500 & Only disconnections & Low decrease \\
5th & 500 & 445 & 575 & Connections and disconnections & Low decrease \\
6th & 500 & 315 & 530 & Connections and disconnections & High decrease \\
\hline
\end{tabular}

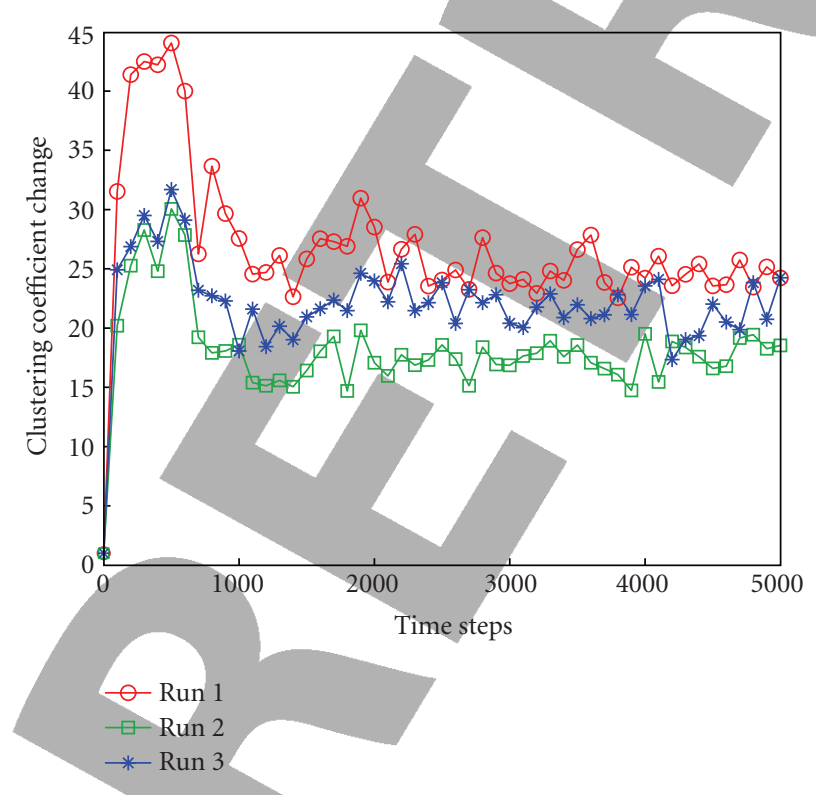

(a)

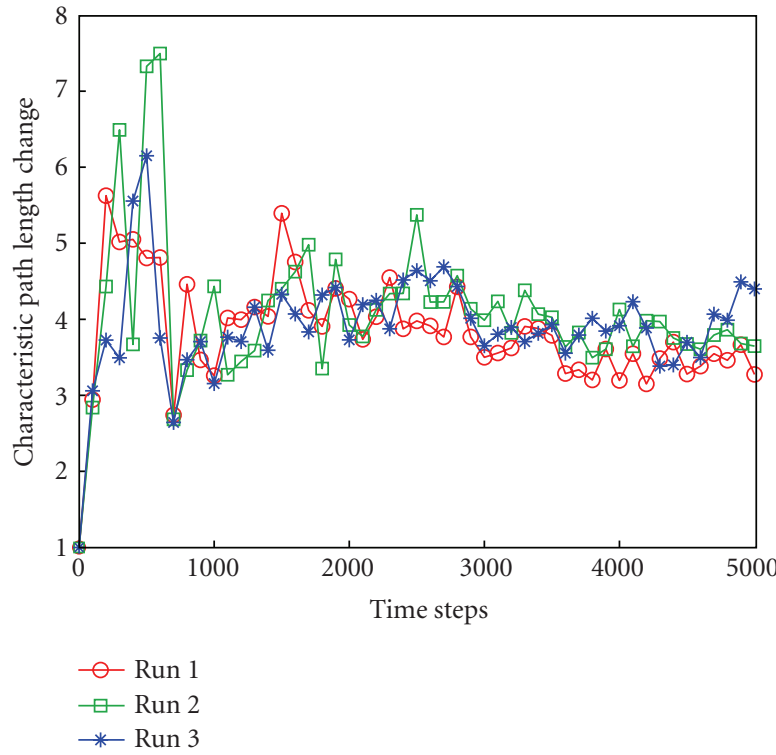

(b)

FIGURE 4: Relative variation of topological metrics using the $3 \mathrm{rd}$ sequence of events. 


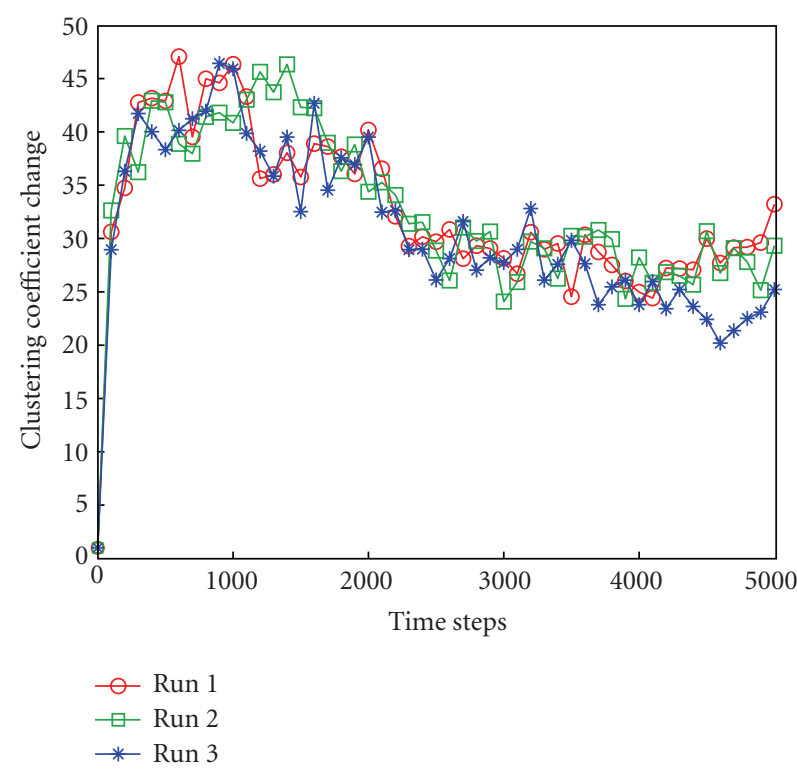

(a)

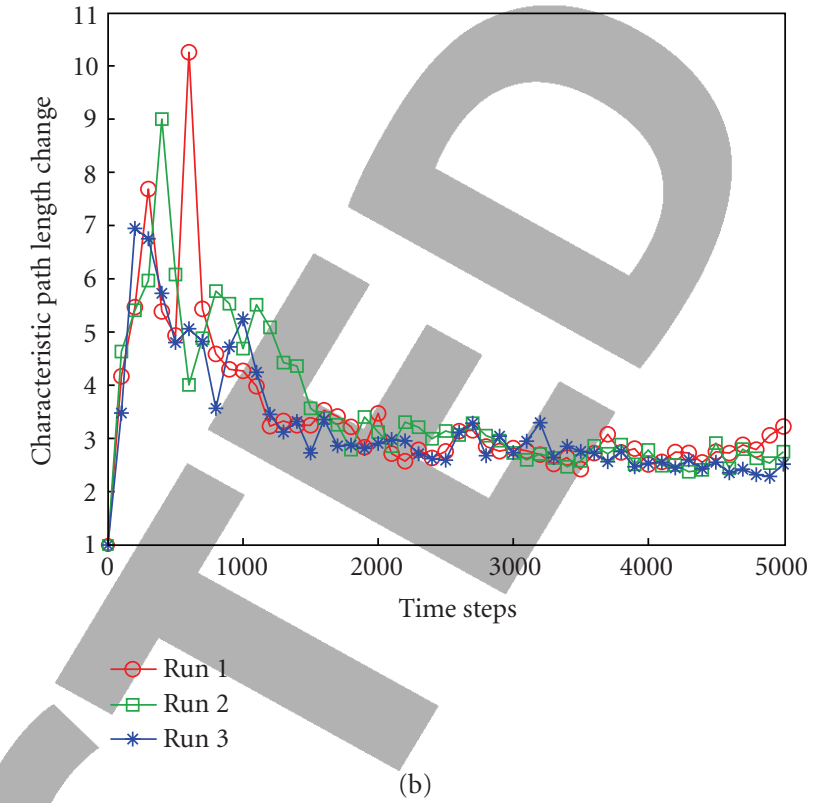

(b)

FIgURE 5: Relative variation of topological metrics using the 6th sequence of events.

in the Internet and broadcasts queries throughout the network using an adaptive routing strategy that dynamically performs local topology adaptations. Modifications in the routing structure are driven by query interactions among neighbors in order to spontaneously create communities of peers that share similar interests; moreover, a small-world network structure can emerge spontaneously thanks to those local interactions. Network traffic cost, and the query efficiency are thus significantly improved as is confirmed by our preliminary experiments.

\section{REFERENCES}

[1] D. Milojicic, V. Kalogeraki, R. Lukose, et al., "Peer-to-peer computing," Tech. Rep. HPL-2002-57, HP Labs, Palo Alto, Calif, USA, 2002.

[2] C-Net News, "Napster among fastest-growing Net technologies," 2000.

[3] Limewire, "The Gnutella protocol specification (ver. 0.4)," http://www9.limewire.com/developer/gnutella_protocol_0.4 .pdf, 2001.

[4] E. Ardizzone, L. Gatani, M. La Cascia, G. Lo Re, and M. Ortolani, "Enhanced $\mathrm{P} 2 \mathrm{P}$ services providing multimedia content," in Proceedings of the 8th IEEE International Symposium on Multimedia (ISM '06), pp. 637-646, San Diego, Calif, USA, December 2006.

[5] I. King, C. H. Ng, and K. C. Sia, "Distributed content-based visual information retrieval system on peer-to-peer networks," ACM Transactions on Information Systems, vol. 22, no. 3, pp. 477-501, 2004.

[6] W. Müller and A. Henrich, "Fast retrieval of high-dimensional feature vectors in P2P networks using compact peer data summaries," in Proceedings of the 5th ACM SIGMM International Workshop on Multimedia Information Retrieval (MIR '03), pp. 79-86, Berkeley, Calif, USA, November 2003.
[7] I. Lee and L, Guan, "Content-based image retrieval with automated relevance feedback over distributed peer-to-peer network," in Proceedings of the IEEE International Symposium on Circuits and Systems (ISCAS '04), vol. 2, pp. 5-8, Vancouver, British Columbia, Canada, May 2004.

[8] M. Kacimi, K. Yétongnon, Y. Ma, and R. Chbeir, "HON-P2P: a cluster-based hybrid overlay network for multimedia object management," in Proceedings of the 11th International Conference on Parallel and Distributed Systems (ICPADS '05), vol. 1, pp. 578-584, Fukuoka, Japan, July 2005.

[9] L.-S. Wu, R. Akavipat, and F. Menczer, "6S: distributing crawling and searching across Web peers," in Proceedings of the IASTED International Conference on Web Technologies, Applications, and Services (WTAS '05), pp. 159-164, Calgary, Alberta, Canada, July 2005.

[10] R. Datta, J. Li, and J. Z. Wang, "Content-based image retrieval: approaches and trends of the new age," in Proceedings of the 7th ACM SIGMM International Workshop on Multimedia Information Retrieval (MIR '05), pp. 253-262, Singapore, November 2005.

[11] A. Del Bimbo, Visual Information Retrieval, Academic Press, New York, NY, USA, 1999.

[12] B. Günsel, A. M. Tekalp, and P. J. L. van Beek, "Content-based access to video objects: temporal segmentation, visual summarization, and feature extraction," Signal Processing, vol. 66, no. 2, pp. 261-280, 1998.

[13] Y. Deng, B. S. Manjunath, C. Kenney, M. S. Moore, and H. Shin, "An efficient color representation for image retrieval," IEEE Transactions on Image Processing, vol. 10, no. 1, pp. 140147, 2001.

[14] E. Hadjidemetriou, M. D. Grossberg, and S. K. Nayar, "Multiresolution histograms and their use for recognition," IEEE Transactions on Pattern Analysis and Machine Intelligence, vol. 26, no. 7, pp. 831-847, 2004.

[15] S. Jeong, C. S. Won, and R. M. Gray, "Image retrieval using color histograms generated by Gauss mixture vector 
quantization," Computer Vision and Image Understanding, vol. 94, no. 1-3, pp. 44-66, 2004.

[16] E. Ardizzone and M. La Cascia, "Automatic video database indexing and retrieval," Multimedia Tools and Applications, vol. 4, no. 1, pp. 29-56, 1997.

[17] B. K. P. Horn and B. G. Schunck, "Determining optical flow," Artificial Intelligence, vol. 17, no. 1-3, pp. 185-203, 1981.

[18] D. J. Watts and S. H. Strogatz, "Collective dynamics of 'smallworld' networks,” Nature, vol. 393, no. 6684, pp. 440-442, 1998.

[19] B. H. Bloom, "Space/time trade-offs in hash coding with allowable errors," Communications of the ACM, vol. 13, no. 7, pp. 422-426, 1970.

[20] J. MacQueen, "Some methods for classification and analysis of multivariate data," in Proceedings of 5th Berkeley Symposium on Mathematical Statistics and Probability, vol. 1, pp. 281-297, Berkeley, Calif, USA, 1967.

[21] M. Halkidi, Y. Batistakis, and M. Vazirgiannis, "On clustering validation techniques," Journal of Intelligent Information Systems, vol. 17, no. 2-3, pp. 107-145, 2001.

[22] N. R. Pal and J. C. Bezdek, "On cluster validity for the fuzzy c-means model," IEEE Transactions on Fuzzy Systems, vol. 3, no. 3, pp. 370-379, 1995.

[23] C. Ordonez, "Clustering binary data streams with K-means," in Proceedings of the 8th ACM SIGMOD Workshop on Research Issues in Data Mining and Knowledge Discovery (DMKD '03), pp. 12-19, San Diego, Calif, USA, June 2003.

[24] S. Kirkpatrick, C. D. Gelatt Jr., and M. Vecchi, "Optimization by simulated annealing," Science, vol. 220, no. 4598, pp. $671-$ 680, 1983.

[25] M. Ripeanu, A. Iamnitchi, and I. Foster, "Mapping the Gnutella network," IEEE Internet Computing, vol. 6, no. 1, pp. 50-57, 2002.

[26] S. Saroiu, P. K. Gummadi, and S. D. Gribble, "Measurement study of peer-to-peer file sharing systems," in Multimedia Computing and Networking, vol. 4673 of Proceedings of SPIE, pp. 156-170, San Jose, Calif, USA, January 2002.

[27] S. Sen and J. Wang, "Analyzing peer-to-peer traffic across large networks," IEEE/ACM Transactions on Networking, vol. 12, no. 2, pp. 219-232, 2004.

[28] L. Gatani, G. Lo Re, and L. Noto, "Efficient query routing in peer-to-peer networks," in Proceedings of the 3rd International Conference on Information Technology: Research and Education (ITRE '05), pp. 393-397, Hsinchu, Taiwan, June 2005.

[29] E. P. Markatos, "Tracing a large-scale peer-to-peer system: an hour in the life of Gnutella," in Proceedings of the 2nd IEEE/ACM International Symposium on Cluster Computing and the Grid (CCGrid '02), pp. 65-74, Berlin, Germany, May 2002.

[30] C. Faloutsos, R. Barber, M. Flickner, et al., "Efficient and effective querying by image content," Journal of Intelligent Information Systems, vol. 3, no. 3-4, pp. 231-262, 1994.

[31] R. W. Picard, “Computer learning of subjectivity," ACM Computing Surveys, vol. 27, no. 4, pp. 621-623, 1995.

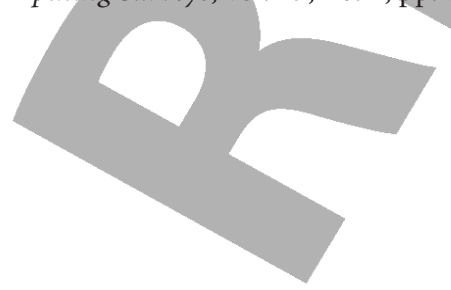



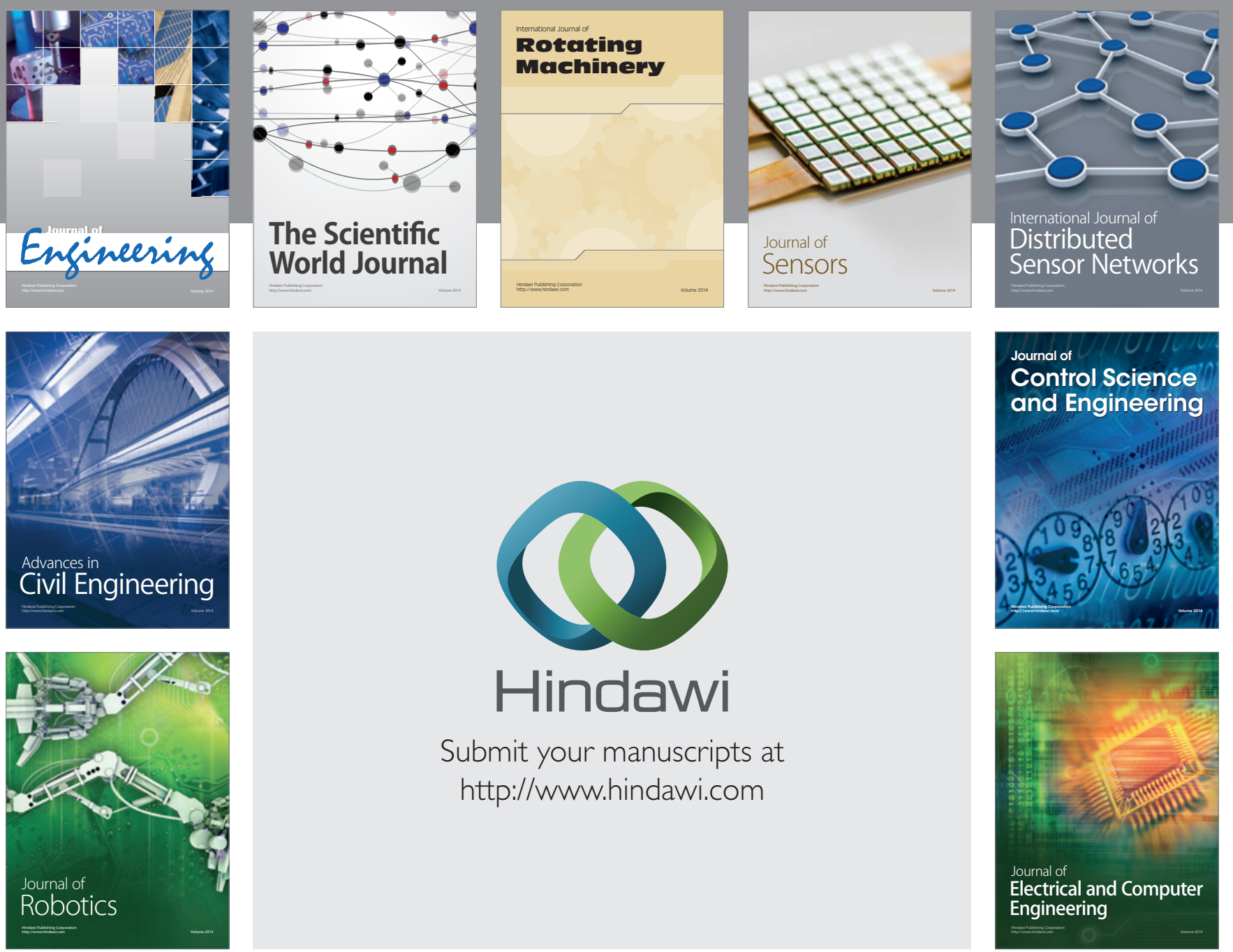

Submit your manuscripts at

http://www.hindawi.com
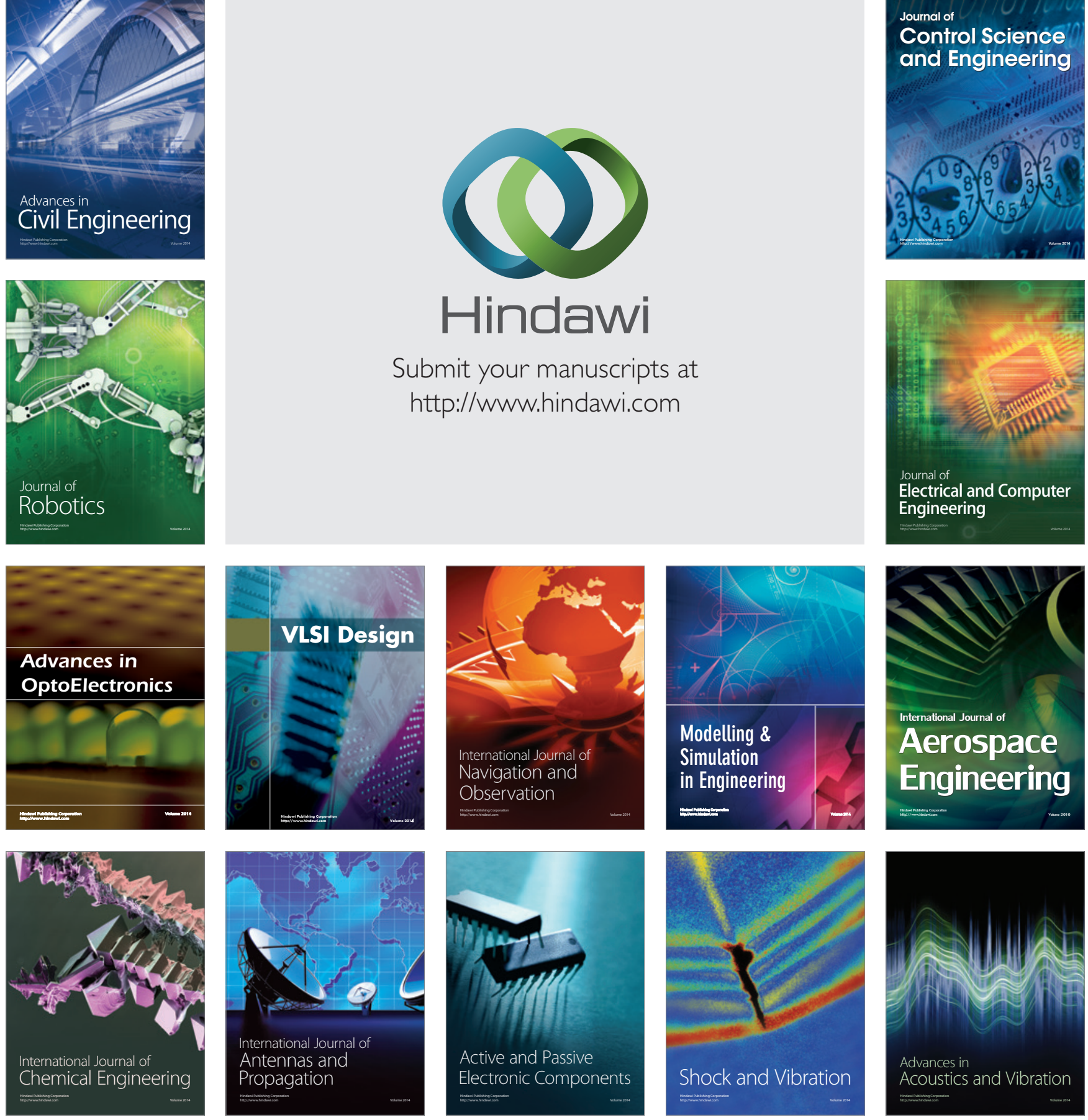